\title{
Desempeño productivo y costos de granjas porcinas con diferentes protocolos de vacunación al virus del PRRS
}

Elizabeth Araceli Quezada-Fraide ${ }^{\mathrm{a}}$

Claudia Giovanna Peñuelas-Rivas ${ }^{b}$

Frida Saraí Moysén-Albarrán $^{c}$

María Elena Trujillo-Ortega ${ }^{\mathrm{d}}$

Francisco Ernesto Martínez-Castañeda ${ }^{\mathrm{c} *}$

${ }^{a}$ Universidad Nacional Autónoma de México. Facultad de Estudios Superiores Cuautitlán. Departamento de Ciencias Pecuarias. México.

${ }^{\mathrm{b}}$ Kansas Smith Farms. EE.UU.

c Universidad Autónoma del Estado de México. Instituto de Ciencias Agropecuarias y Rurales. México.

${ }^{\mathrm{d}}$ Universidad Nacional Autónoma de México. Facultad de Medicina Veterinaria y Zootecnia. Ciudad de México, México.

*Autor de correspondencia: femartinezc@uaemex.mx

\section{Resumen:}

Con el objetivo de evaluar el desempeño productivo y los costos de producción por lechón destetado y cerdo finalizado en granjas porcinas con diferentes protocolos de vacunación al virus del PRRS en condiciones de campo, se analizaron los indicadores total de lechones nacidos, nacidos vivos, mortinatos, destetados, pesos al nacimiento y al destete, días de engorda y peso final así como, los costos por lechón destetado y cerdo finalizado, de dos granjas en régimen de semitecnificación: a) Protocolo 1 (P1), granja con vacunación a reproductoras y a lechones; y b) Protocolo 2 (P2), vacunación únicamente a cerdas 
reproductoras. Los indicadores productivos hasta el destete se evaluaron con un Diseño de Medidas repetidas en el tiempo y los de engorda con un Análisis de varianza con comparación de medias. Los costos se determinaron utilizando la fórmula general de costos. Fue posible observar diferencias en los lechones nacidos totales y momias $(\mathrm{P}<0.05)$ a favor del $\mathrm{P} 2$, de lechones destetados, pesos al nacimiento y al destete a favor del P1 y sin diferencia en el resto de variables. Los cerdos finalizados en P1 tuvieron 12 días más de engorda y un peso final de $3.13 \mathrm{~kg}$ más que P2. Los costos por lechón destetado fueron de \$389.55 y \$424.25 Pesos Mexicanos, y el costo medio por día de engorda fueron de $\$ 10.01$ y $\$ 11.43$, respectivamente.

Palabras clave: Productividad, Costos, Cerdos, Vacunación, PRRS.

Recibido: 20/05/2019

Aceptado: 08/01/2020

\section{Introducción}

El virus del Síndrome reproductivo y respiratorio porcino (PRRSV) del cerdo ha causado significativas pérdidas económicas en la industria porcina en todo el mundo ${ }^{(1)}$. Las pérdidas van desde 75 mil euros en una granja de mil cerdas con una infección "ligera", hasta pérdidas de 698 mil euros ${ }^{(2)}$ y 664 millones de dólares al año en Estados Unidos ${ }^{(3)}$. Es una enfermedad causada por un Arterovirus que emergió a finales de la década de los años ochenta en los EE.UU. ${ }^{(4)}$ y posteriormente en Europa, se dispersó rápidamente y se volvió enzóotica en la población de cerdos del mundo entero ${ }^{(5)}$. La enfermedad se presenta con una gran variedad de signos que reflejan la virulencia de la cepa y que se relacionan con la etapa fisiológica de los animales, su estado inmunológico y la presencia de otras enfermedades ${ }^{(5,6)}$. El cuadro se presenta en dos fases; la primera tiene una duración aproximada de dos semanas, se caracteriza por una viremia aguda que causa anorexia y letargia, así como pirexia, taquipnea y disnea e hiperemia cutánea con extremidades cianóticas. La segunda fase, que puede iniciar antes que la primera se haya completado y puede durar hasta cuatro meses, se caracteriza por la falla reproductiva, principalmente en las cerdas que fueron infectadas durante su tercer tercio de gestación ${ }^{(5)}$, mientras que genera un cuadro respiratorio en cerdos en crecimiento ${ }^{(7)}$.

La enfermedad es causada por un virus RNA del cual existen dos variedades; cepas clásicas (C-PRRSV) y cepas de alta virulencia (HP-PRRSV) ${ }^{(8)}$. También se clasifica de acuerdo con sus variaciones genéticas y diferencias antigénicas en dos tipos; PRRSV-1, el tipo europeo y PRRSV-2, el tipo norteamericano ${ }^{(9)}$. La salud de los animales se puede complicar cuando el 
virus se asocia con otros patógenos como: Circovirus porcino tipo II (PCVII), Pasteurella multocida, Haemophilus parasuis, Bordetella bronchiseptica y Micoplasmas ${ }^{(10,11,12)}$.

Luego de producido el brote, la producción de la granja tiende a mejorar paulatinamente (de 4 a 6 meses), no alcanzando los niveles de producción anteriores al brote. Por otra parte, al quedar el PRRSV circulando, la granja queda expuesta a rebrotes de la enfermedad y a la persistencia del virus en la piara ${ }^{(12)}$.

Los resultados de las intervenciones para reducir el costo de la enfermedad, principalmente en las etapas de engorda han sido positivas, pero dejan y causan mayores pérdidas en la crianza. En 2005, las pérdidas en la piara reproductora de Estados Unidos fueron el $12 \%$ del costo total del PRRS, mientras que, en 2011, el costo en el hato reproductor ascendió a $45 \%{ }^{(3)}$. En este periodo se han implementado diferentes protocolos de intervención que incluyen vacunación, despoblación, protocolos de bioseguridad ${ }^{(13)}$ entre otros.

El impacto negativo del PRRS en el margen económico por cerdo producido ha estimulado los esfuerzos para controlar y eventualmente erradicar la enfermedad. El control del virus de PRRS descansa en aspectos como diagnóstico temprano y monitoreo, bioseguridad, manejo del hato e inmunización ${ }^{(14)}$, sin embargo, estos métodos estándar de control no han sido efectivos toda vez que las vacunas no reducen la prevalencia de la enfermedad y muchos productores tienen que despoblar después de un brote ${ }^{(15)}$.

El PRRS es un modelo hospedero/virus en el cual la enfermedad se determina por la patogenicidad del virus, la susceptibilidad del hato reproductor y el fenotipo, la presión de co-infecciones bacterianas y las condiciones ambientales ${ }^{(16)}$.

Por lo anterior, el objetivo del presente trabajo fue evaluar el desempeño productivo y los costos de producción por lechón destetado y cerdo finalizado en granjas porcinas con dos protocolos de vacunación al virus del PRRS para determinar la eficacia de los mismos y cuál de estos ofrece al productor, mejores indicadores productivos y económicos.

\section{Material y métodos}

El estudio se realizó en dos granjas, que, de acuerdo con la clasificación general tecnológica de la SAGARPA, corresponden a granjas semi-tecnificadas. Las granjas se localizan en el Altiplano. Una en el estado de Hidalgo con un clima templado seco, temperatura media anual de $14{ }^{\circ} \mathrm{C}$ y precipitación pluvial anual de alrededor de $610 \mathrm{~mm}$. La otra en el Estado de México con un clima templado semi-seco, temperatura media anual de 16 a $17{ }^{\circ} \mathrm{C}$ y precipitación pluvial anual de alrededor de 600. El número de cerdas reproductoras fue de 480 y 180 respectivamente. Los datos analizados se tomaron de los registros individuales por 
cerda. El periodo fue desde su primer parto hasta el último registrado, con corte a septiembre de 2017. en un lapso de su vida productiva, siendo el último dato registrado los partos del primer semestre de 2017.

Los indicadores técnicos analizados fueron: lechones nacidos totales, lechones nacidos vivos, lechones nacidos muertos, momias, lechones destetados, peso de los lechones al nacimiento, peso de los lechones al destete, edad de los cerdos al rastro, peso de los cerdos al rastro. Las granjas tienen un diagnóstico positivo a PRRS desde 2003 y se implementa en ambas un calendario de vacunación. En el protocolo de vacunación 1 (P1) se consideró a la granja que vacuna a las cerdas reproductoras y a los lechones a los 21 días de edad, aproximadamente. En el protocolo de vacunación 2 (P2), se consideró a la granja que solo vacuna a las cerdas reproductoras. La vacunación de las cerdas reproductoras en ambas granjas es cada cuatro meses en "sábana". Las dos utilizan una vacuna contra PRRS con virus vivo modificado y una dosis por animal de $2 \mathrm{ml}$.

Ambas granjas tienen similar esquema de bioseguridad, genética y alimentación.

Los análisis de costos se realizaron utilizando la modificación a la fórmula general de costos de Muñoz y Rouco ${ }^{(17)}$.

$\mathrm{TC}=\mathrm{F}+\mathrm{V}$; donde: $\mathrm{TC}=$ costo de lechón destetado, $\mathrm{F}=$ costos fijos y $\mathrm{V}=$ costos variables.

Los costos fijos se formaron por: $\mathrm{F}=\mathrm{L}+\mathrm{S}+\mathrm{Co}+\mathrm{R}+\mathrm{A}+\mathrm{Fi}+\mathrm{CO}+\mathrm{Ot}<$, donde: $\mathrm{L}=$ costos laborales, $\mathrm{S}=$ costos de suministros, $\mathrm{Co}=$ costos de energía y combustible, $\mathrm{R}=$ reparación y mantenimiento, $\mathrm{A}=$ amortización de los activos fijos, $\mathrm{CO}=$ costos de oportunidad y $\mathrm{Ot}=\mathrm{otros}$ costos menores.

Los costos variable se conformaron por las partidas: $\mathrm{V}=((\mathrm{AR}+\mathrm{AM}+\mathrm{AV}+\mathrm{AMV}+\mathrm{AL}+\mathrm{M}+\mathrm{T}+\mathrm{CO} /(\mathrm{TOTCER} * \mathrm{~W})) * \mathrm{z} ; \quad$ donde: $\mathrm{AR}=$ costos de amortización de los reproductores, $\mathrm{AM}=$ alimentación de las cerdas, $\mathrm{AMV}=$ alimentación del verraco, $\mathrm{AV}=$ amortización del verraco, $\mathrm{AL}=$ alimentación de los lechones, $\mathrm{M}=$ medicaciones, $\mathrm{T}=$ transporte, $\mathrm{CO}=$ costos de oportunidad, TOTCER= número total de cerdas en la explotación, $\mathrm{W}=$ Factor de ponderación en virtud de que todos los costos variables se referirán a la unidad de producción de un lechón comercial y z=número de lechones destetados.

La depreciación de los animales reproductores se calculó de la siguiente manera: $\mathrm{AR}=(\mathrm{PH}-(\mathrm{PD}-(1-\mathrm{MORR}))) /(\mathrm{PARM} / \mathrm{PAR})-\mathrm{REP} ;$ donde: $\mathrm{PH}=$ precio de compra de la cerda, $\mathrm{PD}=$ precio de desecho de la cerda, $\mathrm{MORR}=$ mortalidad de reproductoras expresado en porcentaje, $\mathrm{PARM}=$ número promedio de partos de las cerdas, $\mathrm{PAR}=$ número de partos por cerda y año, y REP=reemplazos de reproductoras. 
El promedio de nacimientos por hato reproductor puede ser calculado en cualquier momento de la producción, sin importar la etapa fisiológica en que se encuentren las cerdas.

PARM $=\sum(\mathrm{CER} * \mathrm{n}) / \mathrm{TOTCER}$; donde: $\mathrm{CER}=$ el número de cerdas y $\mathrm{n}=$ número de parto. $\mathrm{PAR}=365 /(114,5+\mathrm{LAC}+\mathrm{INT}) *(1-\mathrm{NAB}+\mathrm{VAC} / \mathrm{CUB})), \mathrm{LAC}=$ duración de la lactancia, $\mathrm{INT}=$ intervalo destete-cubrición fértil, $\mathrm{NAB}=$ número total de abortos, $\mathrm{VAC}=$ número de cerdas vacías, $\mathrm{CUB}=$ número de cubriciones realizadas. A su vez, INT está formado por la suma de los intervalos destete primera cubrición (INT1), p. 100 de primeras repeticiones*21 (INT2), p. 100 segundas repeticiones*42 (INT3), p. 100 de terceras repeticiones*63 (INT4) y p. 100 de repeticiones acíclicas días medios de aparición.

REP=PAR/PARM y el factor de ponderación es: $\mathrm{w}=\mathrm{PAR} * \mathrm{VIV} *(1$ - MOR $) *(1-\mathrm{MORT})$; donde: $\mathrm{PAR}=$ número de partos por cerda y año, $\mathrm{VIV}=$ lechones nacidos vivos por parto, $\mathrm{MOR}=$ mortalidad en lactación, $\mathrm{MORT}=$ mortalidad en transición destete-lechón comercial expresada en puntos porcentuales.

Para el cálculo de costos de cerdo cebado las fórmulas son:

$\mathrm{F}=\mathrm{F}=\mathrm{L}+\mathrm{S}+\mathrm{Co}+\mathrm{R}+\mathrm{A}+\mathrm{Fi}+\mathrm{CO}+\mathrm{Ot}$, aplicable para el proceso de engorda $\mathrm{y}$ $\mathrm{V}=((\mathrm{M}+\mathrm{AL}+\mathrm{CO}) / \mathrm{w})^{*} \mathrm{z}$, aplicable al proceso de engorda, donde $\mathrm{z}$ es el número de lechones engordados.

Para determinar las diferencias en productividad de las cerdas por parto (paridad) y por tipo de protocolo de vacunación, se utilizó un diseño de medidas repetidas en el tiempo. Se determinó la mejor estructura de covarianza y para determinar la significancia se utilizó una prueba de Tuckey ajustada ${ }^{(18)}$. Por su parte, para los datos productivos de la engorda, se utilizó un análisis de varianza para las variables días a venta y peso al sacrificio.

Para determinar las diferencias en ingresos, se utilizó como medida 1 lechón destetado y 1 kilogramo de cerdo engordado. El precio para el cálculo fue de $\$ 28.00 \mathrm{M} / \mathrm{N}$. En el caso de el lechón destetado se utilizó un valor medio de $\$ 800.00 \mathrm{M} / \mathrm{N}$.

\section{Resultados}

Las dos granjas analizadas presentaron similares niveles tecnológicos, de genética de los animales, de alimentación, de manejo productivo y sanitario, a excepción de los protocolos de vacunación contra PRRS. El Cuadro 1 resume el desempeño productivo de las cerdas reproductoras y sus lechones entre protocolos de vacunación. Destaca que a pesar de que el Protocolo 2 ( $\mathrm{P} 2)$ fue mejor en el número de lechones nacidos totales $(P<0.05)$ el número de lechones destetados fue favorable $(P<0.05)$ para el Protocolo $1(\mathrm{P} 1)$. 
Cuadro 1: Comparación de resultados del protocolo de vacunación

\begin{tabular}{lccl}
\hline & \multicolumn{2}{l}{ Protocolo } & \\
\cline { 2 - 3 } & $\mathbf{1}(\mathbf{n = 1 6 5 8})$ & $\mathbf{2}(\mathbf{n = 9 7 2})$ & \\
\hline Lechones nacidos totales & $10.88 \pm 0.10$ & $11.43 \pm 0.12$ & $P<0.0006$ \\
Lechones nacidos vivos & $10.13 \pm 0.13$ & $10.08 \pm 0.15$ & $P>0.05$ \\
Mortinatos & $0.50 \pm 0.04$ & $1.00 \pm 0.12$ & $P>0.05$ \\
Momias & $0.34 \pm 0.03$ & $0.50 \pm 0.03$ & $P<0.0001$ \\
Lechones destetados & $8.94 \pm 0.08$ & $8.31 \pm 0.09$ & $P<0.0001$ \\
Peso camada al & $14.11 \pm 0.13$ & $12.44 \pm 0.10$ & $P<0.0001$ \\
nacimiento & $52.33 \pm 0.52$ & $49.53 \pm 0.59$ & $P<0.0005$ \\
Peso camada al destete & &
\end{tabular}

En este sentido, el número de lechones nacidos totales por paridad solo fue diferente en cerdas en su primer parto $(P<0.05)$ con valores de $10.14 \pm 0.11$ y $11.28 \pm 0.16$ para $\operatorname{los}$ protocolos 1 y 2 , respectivamente.

Con respecto a las paridades (Cuadros 2 y 3), se observaron diferencias en el número de momias en la paridad uno $(0.36 \pm 0.03$ y $0.66 \pm 0.05)$ y paridad cinco $(0.36 \pm 0.03$ y $0.66 \pm$ 0.05); el número de lechones destetados en las paridades dos $(9.19 \pm 0.11 \mathrm{y} 8.01 \pm 0.14) \mathrm{y}$ paridad tres $(9.30 \pm 0.13$ y $7.89 \pm 0.17)$ a favor del P1. En relación con los pesos de la camada al nacimiento, el desempeño fue mejor para P1 en las paridades uno a la cinco. El peso de la camada al destete, sin embargo, solo fue diferente, a favor de P1 en las paridades una a la tres.

Cuadro 2: Desempeño reproductivo en el tiempo (paridad 1 a 4)

\begin{tabular}{lll|ll|ll|ll}
\hline \multicolumn{6}{l}{ Número de paridad } \\
\hline & $\mathbf{1}$ & & $\mathbf{2}$ & & $\mathbf{3}$ & & $\mathbf{4}$ & \\
\cline { 2 - 8 } $\mathrm{T}$ & 1 & 2 & 1 & 2 & 1 & 2 & 1 & 2 \\
& $(\mathrm{n}=493)$ & $(\mathrm{n}=261)$ & $(\mathrm{n}=401)$ & $(\mathrm{n}=204)$ & $(\mathrm{n}=284)$ & $(\mathrm{n}=160)$ & $(\mathrm{n}=208)$ & $(\mathrm{n}=125)$ \\
\hline $\mathrm{LT}$ & $10.14 \pm 0$ & $11.28 \pm 0$ & $10.84 \pm 0$ & $10.86 \pm 0$ & $11.38 \pm 0$ & $11.54 \pm 0$ & $11.56 \pm 0$ & $11.77 \pm 0$ \\
& .11 & .16 & .16 & .18 & .15 & .20 & .17 & .23 \\
& $P<0.0001$ & & $P>0.05$ & & $P>0.05$ & & $P>0.05$ & \\
$\mathrm{LV}$ & $9.39 \pm 0$. & $9.65 \pm 0$. & $10.44 \pm 0$ & $9.59 \pm 0$. & $10.57 \pm 0$ & $10.53 \pm 0$ & $10.56 \pm 0$ & $10.34 \pm 0$ \\
& 17 & 23 & .19 & 26 & .22 & .29 & .26 & .33 \\
& $P>0.05$ & & $P>0.05$ & & $P>0.05$ & & $P>0.05$ & \\
$\mathrm{MR}$ & $0.38 \pm 0$. & $0.93 \pm 0$. & $0.31 \pm 0$. & $0.81 \pm 0$. & $0.46 \pm 0$. & $1.12 \pm 0$. & $0.46 \pm 0$. & $0.95 \pm 0$. \\
& 03 & 07 & 03 & 12 & 06 & 19 & 05 & 09 \\
& $P>0.05$ & & $P>0.05$ & & $P>0.05$ & & $P>0.05$ &
\end{tabular}




\begin{tabular}{lll|ll|ll|ll} 
MM & $0.36 \pm 0$. & $0.66 \pm 0$. & $0.32 \pm 0$. & $0.43 \pm 0$. & $0.38 \pm 0$. & $0.53 \pm 0$. & $0.51 \pm 0$. & $0.55 \pm 0$. \\
& 03 & 05 & 04 & 05 & 04 & 06 & 05 & 07 \\
& $P<0.0001$ & & $P>0.05$ & & $P>0.05$ & & $P>0.05$ & \\
LD & $8.70 \pm 0$. & $8.27 \pm 0$. & $9.19 \pm 0$. & $8.01 \pm 0$. & $9.30 \pm 0$. & $7.89 \pm 0$. & $9.23 \pm 0$. & $8.46 \pm 0$. \\
& 10 & 14 & 11 & 14 & 13 & 17 & 15 & 20 \\
& $P>0.05$ & & $P<0.0001$ & & $P<0.0001$ & & $P>0.05$ & \\
$P N$ & $13.55 \pm 0$ & $12.49 \pm 0$ & $14.70 \pm 0$ & $12.40 \pm 0$ & $14.77 \pm 0$ & $12.79 \pm 0$ & $14.69 \pm 0$ & $12.82 \pm 0$ \\
& .14 & .20 & .16 & .22 & .19 & .25 & .21 & .28 \\
& $P<0.0001$ & & $P<0.0001$ & & $P<0.0001$ & & $P<0.0001$ \\
PD & $51.80 \pm 0$ & $46.21 \pm 0$ & $55.18 \pm 0$ & $46.34 \pm 0$ & $55.47 \pm 0$ & $46.94 \pm 1$ & $54.36 \pm 0$ & $50.39 \pm 1$ \\
& .59 & .81 & .66 & .92 & .78 & .03 & .90 & .16 \\
& $P<0.0001$ & & $P<0.0001$ & $P<0.0001$ & & $P>0.05$ &
\end{tabular}

$\mathrm{T}=$ tratamientos; $\mathrm{LT}=$ lechones nacidos totales $\mathrm{LV}=$ lechones nacidos vivos; $\mathrm{MR}=$ mortinatos; $\mathrm{MM}=$ momias; $\mathrm{LD}=$ lechones destetados; $\mathrm{PN}=$ peso de la camada al nacimiento; $\mathrm{PD}=$ peso de la camada al destete.

Cuadro 3: Desempeño reproductivo en el tiempo (paridad 5 a 7)

\begin{tabular}{lll|ll|ll}
\hline \multicolumn{5}{l}{ Número de paridad } \\
\hline & $\mathbf{5}$ & & $\mathbf{6}$ & & $\mathbf{7}$ & \\
$\mathrm{nyynyyy} \mathrm{T}$ & $1(\mathrm{n}=144)$ & $2(\mathrm{n}=98)$ & $1(\mathrm{n}=93)$ & $2(\mathrm{n}=76)$ & $1(\mathrm{n}=34)$ & $2(\mathrm{n}=48)$ \\
\hline LNT & $11.23 \pm 0.21$ & $11.71 \pm 0.26$ & $10.90 \pm 0.26$ & $11.70 \pm 0.29$ & $10.10 \pm 0.42$ & $11.29 \pm 0.36$ \\
& $P>0.05$ & & $P>0.05$ & & $P>0.05$ & \\
LNV & $10.24 \pm 0.31$ & $10.40 \pm 0.38$ & $10.24 \pm 0.39$ & $10.05 \pm 0.43$ & $9.20 \pm 0.62$ & $9.99 \pm 0.53$ \\
& $P>0.05$ & & $P>0.05$ & & $P>0.05$ & \\
MOR & $0.47 \pm 0.06$ & $1.08 \pm 0.12$ & $0.53 \pm 0.08$ & $1.13 \pm 0.15$ & $0.89 \pm 0.23$ & $0.81 \pm 0.13$ \\
& $P>0.05$ & & $P>0.05$ & & $P>0.05$ & \\
MOM & $0.36 \pm 0.03$ & $0.66 \pm 0.05$ & $0.32 \pm 0.04$ & $0.36 \pm 0.09$ & $0.18 \pm 0.12$ & $0.44 \pm 0.11$ \\
& $P<0.006$ & & $P>0.05$ & & $P>0.05$ & \\
LD & $9.05 \pm 0.18$ & $8.67 \pm 0.22$ & $8.46 \pm 0.23$ & $8.59 \pm 0.25$ & $8.67 \pm 0.37$ & $8.29 \pm 0.31$ \\
& $P>0.05$ & & $P>0.05$ & & $P>0.05$ & \\
PCN & $14.55 \pm 0.26$ & $12.33 \pm 0.31$ & $13.62 \pm 0.32$ & $12.73 \pm 0.36$ & $12.89 \pm 0.53$ & $12.48 \pm 0.44$ \\
& $P<0.0001$ & & $P>0.05$ & & $P>0.05$ & \\
PCD & $52.14 \pm 1.08$ & $52.89 \pm 1.31$ & $48.07 \pm 1.33$ & $51.41 \pm 1.48$ & $49.30 \pm 2.18$ & $52.55 \pm 1.85$ \\
& $P>0.05$ & & $P>0.05$ & & $P>0.05$ & \\
\hline
\end{tabular}

$\mathrm{T}=$ tratamientos; $\mathrm{LNT}=$ lechones nacidos totales; $\mathrm{LNV}=$ lechones nacidos vivos; $\mathrm{MOR}=$ mortinatos; $\mathrm{MOM}=$ momias; $\mathrm{LD}=$ lechones destetados; $\mathrm{PCN}=$ peso de la camada al nacimiento; $\mathrm{PCD}=$ peso de la camada al destete.

El número de lechones nacidos totales en la granja con el P1, registró un incremento en las paridades 2, 3, 4 y 5, con respecto al parto $1(P<0.05)$. Los valores fueron: $10.14 \pm 0.11$, $10.84 \pm 0.16,11.38 \pm 0.15,11.56 \pm 0.17$ y $11.23 \pm 0.21$, respectivamente. En la granja con el P2, no hubo diferencia en el indicador. El número de momias en P1, no presentó 
diferencias, por su parte, el número de momias bajo P2 fue diferente $(P<0.05)$ en los partos 1,6 y 7 . Los valores fueron de: $0.66 \pm 0.05$ (parto uno), $0.43 \pm 0.05,0.36 \pm 0.09$ y $0.44 \pm$ 0.11. El desempeño en lechones destetados para P1 fue diferente en los partos 2 y 3 con respecto a parto 1 , mientras que no se registraron diferencias en este indicador para los cerdos en P2. Los pesos al nacimiento en P1 registró una disminución en los partos 2, 3, 4 y 5 $(P<0.05)$ y sin diferencia en $\mathrm{P} 2$. Finalmente, los pesos de la camada al destete se comportaron de manera regular, con diferencias en los lechones destetados bajo P1 en los partos 2 y 3 , con relación a parto 1 y en $\mathrm{P} 2$, la diferencia se observó en la paridad cinco.

Para el caso de engorda los días a venta registrados fueron de $181.08 \pm 5.01$ y $168.81 \pm 4.81$ para P1 y P2 respectivamente $(P<0.05)$; el peso final fue de: $95.46 \pm 3.27$ y $92.28 \pm 3.93$ $(P<0.05)$, respectivamente.

Los costos de producción por lechón destetado en P1 fue de $\$ 389.55$ pesos y de $\$ 424.25$ para P2. El 93.62\% del total de los costos fueron costos variables en el P1 mientras que, en el P2, los costos variables fueron de $96.27 \%$.

El costo en engorda fue de $\$ 1,812.81$ en $\mathrm{P} 1$ y de $\$ 1,930.07$ en P2. Con estos indicadores productivos, el costo medio por día de engorda fue de \$10.01/día en P1 y \$11.43/día en P2, respectivamente.

El ingreso por lechón destetado fue de $\$ 410.45 \mathrm{M} / \mathrm{N}$ para el P1 y de $\$ 375.75$ en el P2. Así mismo, para un cerdo finalizado, el ingreso fue de $\$ 860.07$ y $653.77 \mathrm{M} / \mathrm{N}$, respectivamente.

\section{Discusión}

Al desglosar los datos por parámetro por granja y por paridad y comparándolos con el conglomerado de producción de $\mathrm{PIC}^{(19)}$ para México, las granjas se encuentran 2.75 y 2.2 lechones nacidos totales menos, en las granjas con P1 y P2, respectivamente. En el número de lechones nacidos vivos la diferencia fue de -2.18 y 2.23 , lechones destetados de -1.95 y -2.58, respectivamente. Estos datos de PIC corresponden al $10 \%$ superior en producción, en términos de producción. Sin embargo, las granjas con P1 y P2, están abajo en 1.21 y 1.26, respectivamente, comparándolos con el $10 \%$ con peor desempeño productivo ${ }^{(20)}$. Igualmente, el peso al destete fue menor en las granjas analizadas: -470 y $-360 \mathrm{~g}$.

Las diferencias en estos resultados en general sugieren que el PRRS al afectar a las reproductoras puede atravesar la placenta alrededor del día 70 de la gestación, provocando que se presenten partos prematuros, por un lado, y por el otro, una mayor cantidad de lechones nacidos muertos y momias. Igualmente, es posible registrar un aumento de mortalidad predestete, significando un menor número de lechones destetados ${ }^{(14,21)}$. Aun 
cuando la literatura menciona que una granja afectada en un lapso de seis meses regresa a una "relativa normalidad"(14), hay algunas granjas donde esta enfermedad se encuentra de forma crónica ${ }^{(21)}$. El virus del PRRS crea sinergia con otros virus o bacterias que pueden ser la causa de mayor mortalidad en la lactancia ${ }^{(22)}$. En un ciclo abierto, el virus del PRRS en granjas inestables puede ser detectado en todos los grupos de cerdos incluyendo a los lechones ${ }^{(16)}$, lo que implica que se pueden presentar resultados adversos causados por el virus, aún incluyendo los protocolos de vacunación.

En estudios donde se incluye la vacunación del virus del PRRS en cerdas multíparas y en cerdas primerizas, se ha demostrado que se confiere una protección inmunológica pasiva a los lechones de hasta 84 días, independientemente de si los lechones son o no vacunados antes de este periodo ${ }^{(23)}$. A pesar de que no se tiene muchos estudios de la protección a mayor edad, estos datos sugieren, susceptibilidad en los animales de engorda, toda vez que no sean inmunizados.

En un estudio realizado en granjas estables con vacunación con virus vivo modificado de PRRS, se encontró, que existen algunas pérdidas y cambios negativos en los indicadores productivos de las cerdas ${ }^{(24)}$. Se registró un ligero incremento en la tasa de mortalidad predestete y sin cambios significativos en la tasa de abortos, pérdidas neonatales, cerdos destetados por ceda e intervalo destete primer servicio.

La vacunación en masa de todo el hato reproductor ha sido reportada como estrategia favorable de protección contra el virus del PRRS, incrementando en un lechón destetado por cerda al año ${ }^{(25)}$; sin embargo, otros estudios sugieren que el cambio es mínimo ${ }^{(24)}$ no hay diferencias significativas ${ }^{(26)}$ e inclusive, se han reportado indicadores $\operatorname{adversos}^{(27)}$.

En cuanto al desempeño en la engorda, tomando como referencia los resultados promedio de PIC para México, la diferencia en edad fue de 18 días más para P1 y 6 días más para P2 y $-23.63 \mathrm{~kg}$ para $\mathrm{P} 1 \mathrm{y}-26.81 \mathrm{~kg}$ para $\mathrm{P} 2$.

El PRRS afecta a los cerdos en engorda produciendo enfermedades de tipo respiratorio con lesiones pulmonares, permitiendo que otras enfermedades virales o bacterianas como la Influenza, Streptococcus suis, Mycoplasma hyopneumoniae, Salmonella cholerasuis, Haemophilus parasuis, Pasteurella multocida, Circovirus porcino, Coronavirus porcino, Actinobacillus pleuroneumoniae ${ }^{(14,22)}$ se asocien con ésta. Estas enfermedades reducen el crecimiento de los cerdos, la ganancia diaria disminuye y aumentan los días al rastro.

Es importante resaltar que las granjas utilizadas en este estudio son granjas estables a PRRS de acuerdo a la Asociación Americana de Veterinarios de cerdos, y estos resultados no son aplicables a granjas libres de PRRS o a granjas PRRS positivas inestables. 
A pesar de las diferencias en diferentes reportes científicos, después de 20 años de vacunación contra el virus de PRRS con vacunas de virus vivo modificado, ésta sigue brindando protección y los resultados han sido confirmados en 35 millones de cerdos a los que se les ha aplicado ${ }^{(28)}$.

\section{Conclusiones e implicaciones}

Como se ha mencionado, estos datos proveen información para alimentar modelos económicos que ayuden a especialistas en producción porcina y a productores, en la toma de decisiones con evidencias de campo, acerca del uso de la vacunación de PRRS como una estrategia preventiva. Si bien no se observaron significancias en las granjas que vacunan a las reproductoras, el costo por lechón destetado fue menor, \$389.55 en el P1 que en el P2 $\$ 424.25$. Para la etapa de engorda o finalización, vacunar tanto a las cerdas reproductoras como a los lechones bajo el P1, tuvo los mejores resultados tanto productivos como económicos.

\section{Agradecimientos y conflicto de intereses}

A los productores por su apoyo y disposición. Al CONACyT por la beca para la realización de los estudios de la MVZ Elizabeth Araceli Quezada Fraide.

No existe ni existió conflicto de interés alguno.

\section{Literatura citada:}

1. Lunney JK, Benfield D, Rowland RRR. Porcine reproductive and respiratory syndrome virus: an update on an emerging and re-emerging viral disease of swine. Virus Res 2010;154:1-6.

2. Nauthes H, Alarcon P, Rushton J, Jolie R, Fiebig K, Jimenez M, Geurts V. Cost of porcine reproductive and respiratory syndrome virus at individual farm level - An economic disease model. Prev Vet Med 2017;142:16-29.

3. Holtkamp DJ, Kliebenstein JB, Neumann EJ, Zimmerman JJ, Rotto HF, Yoder TK, et al. Assessment of the economic impact of porcine reproductive and respiratory syndrome virus on United States pork producers. J Swine Health Prod 2013;21(2):72-84.

4. Keffaber KK. Reproductive failure of unknown etiology. Amer Assoc Swine Pract 1989;1:1-10.

5. Zimmerman JJ, Karriker LA, Ramirez A, Schwartz KJ, Stevenson GW. Diseases of swine. 10th ed. USA: Wiley-Blackwell; 2012. 
6. Wang G, Yu Y, He X, Wang M, Cai X. Zimmerman JJ. Porcine reproductive and respiratory syndrome virus infection of bone marrow: Lesions and pathogenesis. Vir Res 2019;265:20-29.

7. Oh T, Kim H, Park KH, Jeong J, Yang S, Kang I, Chae C. Comparison of four commercial PRRSV MLV vaccines inherds with co-circulation of PRRSV-1 and PRRSV-2. Comp Immunol Microbiol Infect Dis 2019;63:66-73.

8. Wang G, Yu Y, Zhang C, Tu Y, Tong J, Liu Yonggang, et al. Immune responses to modified live virus vaccines developed from classical or highly pathogenic PRRSV following challenge with highly pathogenic PRRSV strain. Dev Comp Immunol 2016;62:1-7.

9. Xie J, Christiaens I, Yang B, Van Breedam W, Cui T, Nauwynck H. Molecular cloning of porcine Siglec-3, Siglec-5 and Siglec-10, and identification of Siglec-10 as an alternative receptor for porcine reproductive and respiratory syndrome virus (PRRSV). J Gen Virol 2017;98:2030-2042.

10. Yin SH, Xiao CT, Gerber PF, Beach NM, Meng XJ, Halbur PG, Opriessnig T. Current porcine circovirus type 2a (PCV2a) or PCV2b infection increases the rate of amino acid mutations of porcine reproductive and respiratory syndrome virus (PRRSV) during serial passages in pigs. Vir Res 2013;178:445-451 .

11. Li J, Wang S, Li C, Wang C, Liu Y, Wang G, et al. Secondary Haemophilus parasuis infection enhances highly pathogenic porcine reproductive and respiratory syndrome virus (HP-PRRSV) infection-mediated inflammatory responses. Vet Microbiol 2017;204:35-42.

12. Willis RW, Zimmerman JJ, Yoon KJ, Swenson SL, McGinley MJ, et al. Porcine reproductive and respiratory syndrome virus: a persistent infection. Vet Microbiol 1997;55,231-240.

13. Silva GS, Corbellini LG, Linharesa DLC, Bakera KL, Holtkamp DJ. Development and validation of a scoring system to assess the relative $\mathrm{T}$ vulnerability of swine breeding herds to the introduction of PRRS virus. Prev Vet Med 2018;160:116-122.

14. Pileri E, Mateu E. Review on the transmission porcine reproductive and respiratory syndrome virus between pigs and farms and impact on vaccination. Vet Res 2016;47:108-121.

15. Prather RS, Whitworth KM, Schommer SK, Wells KD. Genetic engineering alveolar macrophages for host resistance to PRRSV. Vet Micriobiol 2017;209:124-129. 
16. Drigo M, Giacomini E, Lazzaro M, Pasotto D, Bilato D, Rueggeri J, Boniotti MB, Alborali GA, Amadori M. Comparative evaluation of immune responses if swine in PRRS-stable unstable herds. Vet Immunol Immunopatol 2018;200:32-39.

17. Muñoz LA, Rouco YA. Análisis de costos de producción de lechón comercial en explotaciones tipo de la Región de Murcia. Archiv Zoot 1995;44:391-402.

18. Kramer CY. Extension of multiple range tests to group means with unequal numbers of replications. Biometrics 1956;12:307-310.

19. PIC. Análisis de la industria porcina en Latinoamérica. PIC 2017;15:1-20.

20. PigCHAMP Magazine. Benchmark. 2016. USA.

21. Morilla A. Manual para el control de las enfermedades infecciosas de los cerdos. 2a ed. ed. México: Manual Moderno; 2005.

22. Schwartz KJ. Swine disease manual. Third ed, Perry, USA: American Association of Swine Practitioners 2005.

23. Kittiwan N, Yamsakul P, Tadee P, Tadee P, Nuangmek A, Chuammitri P, Patchanee P. Immunological response to porcine reproductive and respiratory syndrome virus in young pigs obtained from a PRRSV-positive exposure status herd in a PRRSV endemic area. Vet Immunol Immunopat 2019;218: 09935.

24. Moura CAA, Johnson C, Baker SR, Holtkamp DJ, Wang C, Linhares DCL. Assessment of immediate production impact following attenuated PRRS type 2 virus vaccination in swine breeding herds. Porc Health Manag 2019;5:13.

25. Linhares DCL, Johnson C, Morrison RB. Economic analysis of immunization strategies for PRRS control. PLoS One. 2015;10(12): e0144265.

26. Eclercy J, Renson P, Lebret A, Hirchaud E, Normand V, Andraud M, et al. A field recombinant strain derived from two type 1 porcine reproductive and respiratory syndrome virus (PRRSV-1) modified live vaccines shows increased viremia and transmission in SPF pigs. Viruses 2019;11(3).

27. Dewey CE, Wilson S, Buck P, Leyenaar JK. The reproductive performance of sows after PRRS vaccination depends on stage of gestation. Prev Vet Med 1999;40(3-4):233-241.

28. Jeong J, Choi K, Kang I, Park C, Chae C. Evaluation of a 20 year old porcine reproductive and respiratory syndrome (PRRS) modified live vaccine (Ingelvac1 PRRS MLV) against two recent type 2 PRRS virus isolates in South Korea. Vet Microb 2016;192:102-109. 